\title{
Pediatric Endoscopic Endonasal Approaches for Skull Base Lesions in the Very Young: Is It Safe and Effective?
}

\author{
${ }^{1}$ Department of Surgery, Division of Otolaryngology, University of \\ California San Diego, San Diego, California, United States \\ 2 Department of Neurosurgery, University of California San Diego, San \\ Diego, California, United States \\ 3 Rady Children's Hospital San Diego, San Diego, California, United States \\ ${ }^{4}$ University of California San Diego School of Medicine, San Diego, \\ California, United States
}

Javan Nation ${ }^{1,3}$ Alexander J. Schupper ${ }^{4}$ Adam Deconde ${ }^{1} \quad$ Michael Levy ${ }^{2,3}$

J Neurol Surg B 2018;79:574-579.

\author{
Address for correspondence Javan Nation, MD, Department of \\ Otolaryngology, Rady Children's Hospital, 3020 Children's Way, MC \\ 5024, San Diego, CA 92123, United States \\ (e-mail: jnation@ucsd.edu).
}

\begin{abstract}
Keywords

- pediatric endoscopic endonasal approach

- expanded endoscopic endonasal approach

- EEA

- pediatric skull base surgery

- surgical complications

Introduction The fully endoscopic expanded endonasal approach (EEA) has been shown to be safe and efficacious in pediatric patients. However, in the very young patient (ages six and under), the anatomical challenge of working through a small nasal corridor is problematic. The ability to repair the skull base and use a nasoseptal flap (NSF) has also been called into question. Here, we review skull base resections using EEA in patients aged 6 years and younger.

Methods A retrospective chart review was conducted on pediatric patients age 6 years and younger who underwent EEA skull base resections over a 3-year period (June 2014 through June 2017).

Results Eight children aged 6 and under with seven tumor pathologies underwent an EEA for the resection of their skull base tumors, with a mean follow-up of 1.45 years. Only chordoma cases required multiple-staged resections. There are no tumor recurrences to date. A high-flow intraoperative cerebrospinal fluid (CSF) leak was encountered in three cases, and there were no postoperative CSF leaks. CSF leaks were effectively repaired with NSFs. One patient suffered from postoperative hypopituitarism, one patient experienced recurrent epistaxis secondary to turbinate destruction by the tumor, and one patient expired 8 months postresection secondary to metastatic disease.

Conclusion In our case series, EEA for skull base tumors in children aged 6 and under was a safe and effective surgical approach. Skull base defects are able to be effectively addressed with typical repairs including NSFs. Larger studies are warranted to further investigate this technique in this pediatric population.
\end{abstract}

\section{Introduction}

The fully endoscopic expanded endonasal approach (EEA) is a technique utilized by neurosurgeons and otolaryngologists to provide minimally invasive access to the ventral skull base. Previous studies have shown that it can be successfully executed in the pediatric population. ${ }^{1-5}$ Compared with the transcranial approach, EEA carries the advantage of no skin incision, craniotomy, or brain retraction, which may lead to less postoperative complications. ${ }^{1}$ When EEA was directly compared with craniotomy, patients who received EEA received a similar extent of resection and decreased rates of neurological injury. ${ }^{6}$ Additionally, EEA has associated lower costs compared with transcranial surgery. ${ }^{7}$ Despite these lower associated complication risks, EEA is associated with the risk of neurovascular injury, which is thought to be in large part due to the anatomically immature skull base in children. ${ }^{8}$ Additionally, the endoscopic endonasal approach is associated received

November 29, 2017

accepted after revision

March 2, 2018

published online

April 30, 2018 (c) 2018 Georg Thieme Verlag KG Stuttgart · New York
DOI https://doi.org/ 10.1055/s-0038-1645854. ISSN 2193-6331. 
with cerebrospinal fluid (CSF) leaks. Pediatric skull base tumor resections are associated with an $8 \%$ CSF leak. ${ }^{1}$ Stapleton et al reviewed specific factors with associated CSF leak risk, and found that the location of the skull base defect and degree of intraoperative CSF flow were associated risk factors. ${ }^{9}$ Vascularized flaps have been identified as preferential over nonvascularized flaps, ${ }^{9}$ particularly in high-flow intraoperative CSF leaks. ${ }^{10}$ Age and clival chordomas have been associated with higher risk of postoperative CSF leaks. ${ }^{9}$

Despite several studies looking at the safety and efficacy of pediatric EEA, there is a lack of literature focusing on the young pediatric patient. The young pediatric patient has unique challenges based on developing anatomy that need to be considered. The piriform aperture is significantly smaller in children aged 6 and under, but is thought to only be an anatomical limit for patients aged 2 and under. ${ }^{8}$ Sphenoid pneumatization has anatomic variations, and begins around age 3 and ends by age $10.8,10$ This study aims to provide further evaluation on EEA in the young pediatric patient, defined as age 6 and younger. We hypothesize that similar to older pediatric patients, younger pediatric patients with skull base tumors may also benefit from EEA.

\section{Methods}

Institutional Review Board approval was obtained to access patient cases for this study. To review the cases of young children who received resection of their tumor by the EEA, we utilized a retrospective chart review. Children aged 6 and under with surgically amenable skull base pathology who underwent EEA were included in this study. Eight patients between June 2014 and June 2017 met the inclusion criteria. Patient age, indication for surgery, tumor pathology, and tumor site were recorded. Surgical data including surgical corridor, whether a staged resection was required, how the corridor was repaired, whether a septal flap and/or lumbar drain was utilized, gross total versus subtotal resection (GTR defined as at least $90 \%$ of tumor debulking, vs. $<90 \%$ for subtotal resection), and the prevalence of intraoperative CSF leak were analyzed. Postoperative CSF leak, optic outcomes, endocrine sequelae, other complications, and follow-up were measured. Pre- and postoperative magnetic resonance imagings were also analyzed for comparison.

\section{Results}

\section{Tumor Characteristics}

- Table 1 includes tumor classification data. Over a 3-year period, eight patients received EEA for the resection of their skull base tumor. Patients were between 1 and 6 years of age (average $=4.29$ years, median $=5$ years). Tumor pathologies included the following: craniopharyngioma, neuroblastoma, Rathke cleft cyst, nasofrontal encephalocele, mesenchymal hamartoma, dermoid cyst, and two chordomas. Five (62.5\%) tumors were located in the sella; one was paracribiform, one infraplanum, and one retrofrontal. The two chordoma cases were both sellar and clival (- Fig. 2). The craniopharyngioma presented with hydrocephalus ( - Fig. 1 ), the Rathke cleft cyst

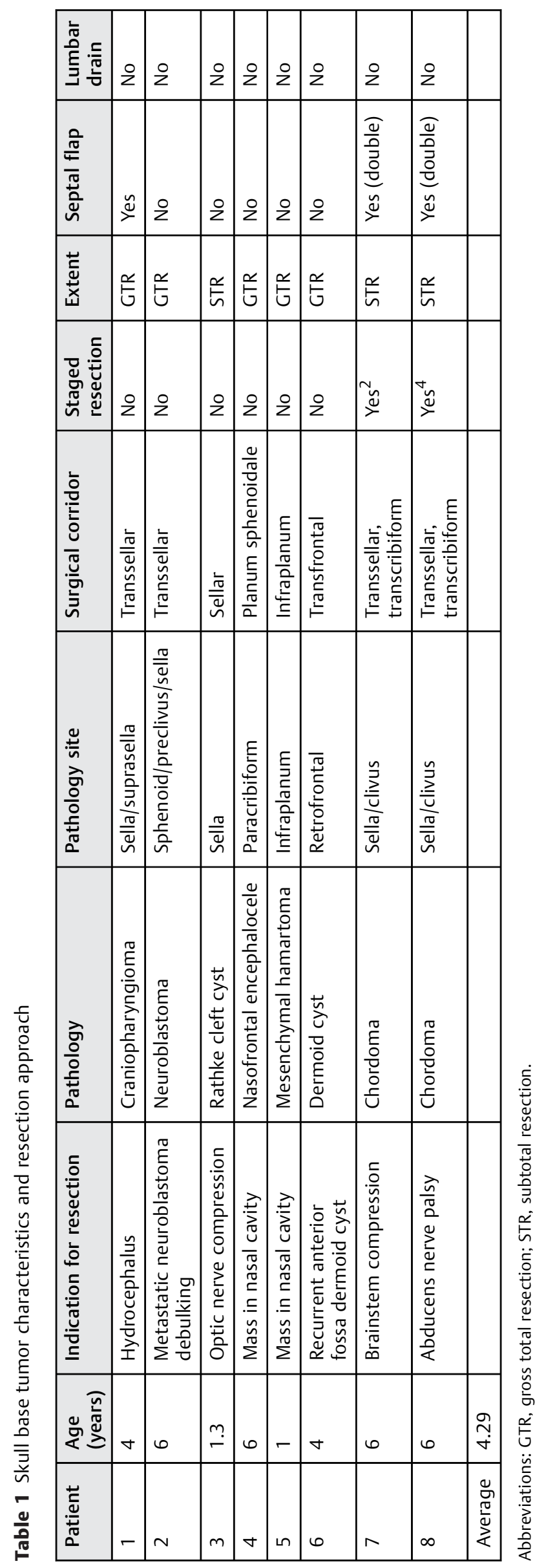


compressed the optic nerve, the nasofrontal encephalocele and mesenchymal hamartoma were found as masses in the nasal cavity, the neuroblastoma was addressed for debulking of metastatic disease, the dermoid cyst was a recurrence in the anterior fossa, and the chordomas presented with an abducens nerve palsy and mass effect causing brainstem compression.

\section{Surgical Approach}

Four of the cases required transsellar approaches (two chordoma cases were also transclival), one sellar, one transfrontal, and the paracribiform tumor was resected via a planum sphenoidale corridor. In all four of the transsellar approaches, the sphenoid sinus was not pneumatized, and drilling was required to access the sella. Six (75\%) cases required repair of the surgical corridor. Four cases used an alloderm underlay with Gelfoam and Merocel for repair, with one of the chordoma cases also requiring Duraseal. The craniopharyngioma case required a fat graft, alloderm underlay, nasoseptal flap (NSF) and Gelfoam, and the Rathke cleft cyst only requiring Gelfoam. A single septal flap was used for the craniopharyngioma case, and double NSFs were utilized for the chordomas. No lumbar drains were used in these cases. GTR was obtained in five (62.5\%) cases. Only the two chordoma cases required staged resections, with one case requiring two stages and the other requiring four.

\section{Complications and Outcomes}

- Table 2 includes patient complication data. Intraoperative CSF leaks occurred in four (50\%) cases, with three cases being high flow and one low flow. Zero patients experienced postoperative CSF leaks. Two (25\%) patients experienced persistent postoperative ophthalmic changes that were present prior to resection, one with slight color asymmetry between left and right fields, anisometropia and unilateral astigmatism, and the other patient having enophthalmos with refractive error. One (12.5\%) patient suffered from postoperative endocrine sequelae with hypopituitarism (associated TSH, thyroid stimulating hormone and antidiuretic hormone deficiencies). Two (25\%) patients had immediate postoperative diabetes insipidus (DI), one of who suffered residual DI. Four patients suffered from other complications, including the craniopharyngeal case with recurrent sphenoid sinus crusting requiring septal revision 1 year postresection, the mesenchymal hamartoma case with recurrent postoperative epistaxis, one of the patients with a chordoma suffered from postoperative velopharyngeal insufficiency, and the child with the neuroblastoma tumor suffered from metastatic disease that was chemotherapy-resistant, ultimately leading to expiration 7 months postresection. Patients followed up with their primary surgeon for an average 1.45 years (range $=0.58-2.5$ years).

\section{Discussion}

The pediatric population has unique challenges in tumor resection for skull base tumors. Anatomically, younger children vary in their anatomy even when compared with older children. The piriform aperture is significantly narrow in younger children, clival pneumatization occurs during early

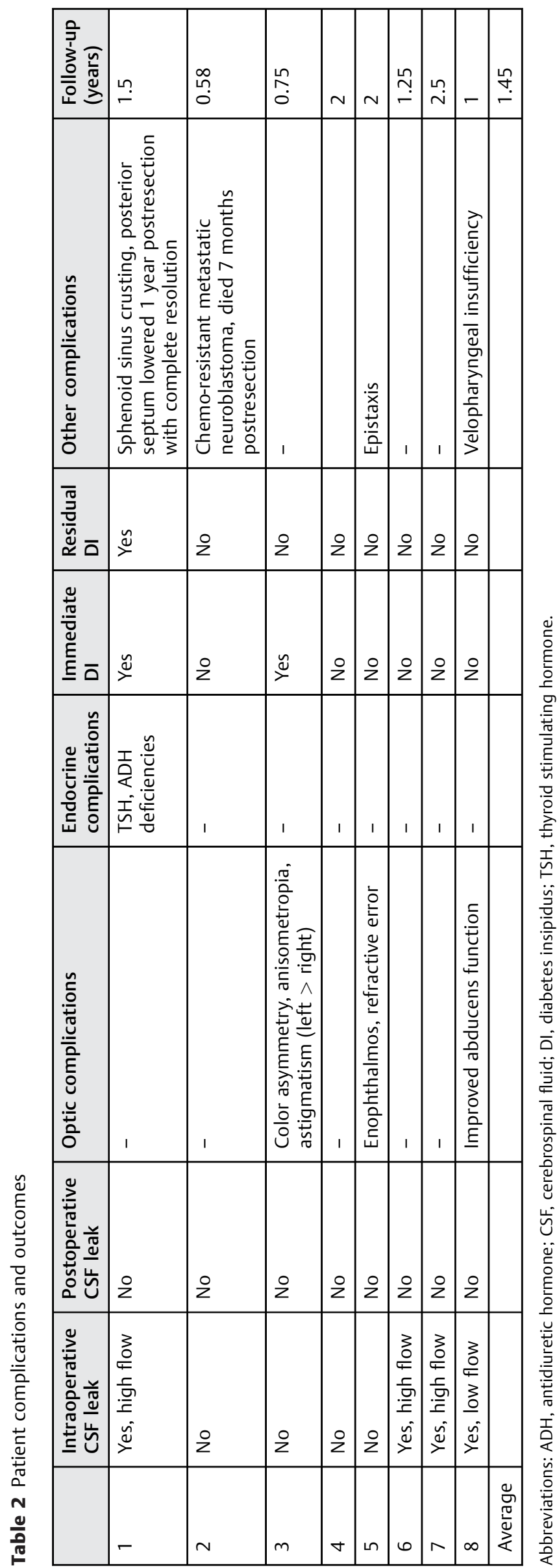




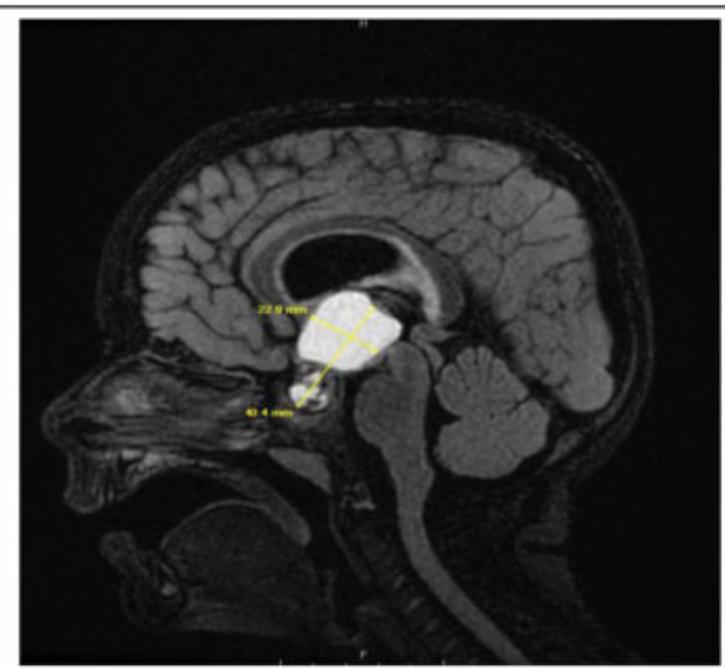

Pre-operative sagittal T2 FLAIR image of a craniopharyngioma in a six year old child. Patient received a transsellar resection, requiring a nasoseptal flap.

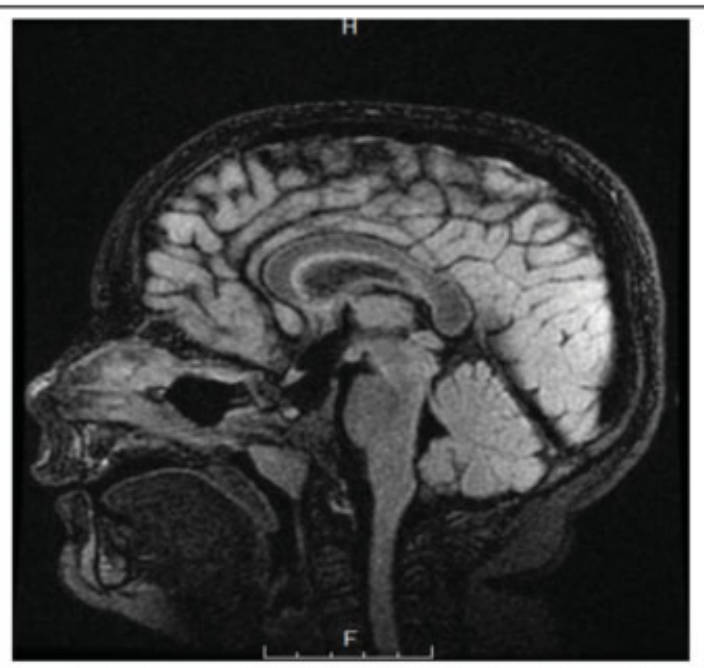

Post-operative sagittal T2 FLAIR section of same patient. A gross total resection of the tumor burden was achieved. The patient did experience postoperative endocrine changes, as well as sphenoid sinus crusting which required septal revision one year post-resection.

Fig. 1 Pre- and postoperative magnetic resonance imaging, Patient 1. (A) Preoperative sagittal T2 fluid-attenuated inversion recovery (FLAIR) image of a craniopharyngioma in a 6-year-old child. Patient received a transsellar resection, requiring a nasoseptal flap. (B) Postoperative sagittal T2 FLAIR section of same patient. A gross total resection of the tumor burden was achieved. The patient did experience postoperative endocrine changes, as well as sphenoid sinus crusting which required septal revision 1 year postresection.

adolescent years, and the intercarotid distances must be taken into account. ${ }^{4}$ Sphenoid sinus pneumatization does not occur until 7 years of age, and the additional challenge of drilling through cancellous bone to expose the landmarks on the face of posterior sphenoid wall is present if very young children. $^{8}$

NSFs in the pediatric population have only recently been added to the literature. In the past 10 years, it was concluded that children aged under 10 years undergoing anterior skull base surgery were not viable candidates for $\mathrm{NSF}^{2}{ }^{2}$ The relationship between age and NSF length to sellar defect ratio has been established, with younger patients having a greater flap to defect ratio, suggesting a feasible reconstruction. ${ }^{11}$ The Purcell et al study and other studies have supported the utility of NSF for suprasellar reconstruction, but use an older pediatric population, with average ages in the teenage years. ${ }^{2,11,12}$ Shah et al found that younger patients had less adequate flap coverage compared with older patients, and that the flap length was less than the transsellar defect length until 6 to 7 years of age. ${ }^{2}$ In this study, it was also determined that in the transsellar/transplanar approach, children aged under 6 years did not have sufficient tissue for a successful flap. Among our patients, a 4-year-old with a craniopharyngioma received a NSF, along with fat grafting and alloderm underlay. Our two patients with chordomas (both age 6 years) received a double NSF (one flap including mucosal septum extending to the caudal septum and the other side extending to the bony cartilaginous junction) which was also repaired with an alloderm underlay, Gelfoam, Duraseal, and Merocel. All three patients experienced intraoperative CSF leaks, with two being high flow and one of the chordoma cases being low flow; however, all patients healed adequately, with no postoperative CSF leaks. The 6-year-old patient with the craniopharyngioma had persistent sphenoid crusting that only resolved after the posterior septum was lowered 1 year after surgery. Despite our small sample size and previously reported anatomical considerations, it may be reasonable to use a NSF on children aged 6 and under with high-flow intraoperative leaks, as we have demonstrated. Preoperative measurements, including the anterior skull base, size of the surgical corridor, and potential NSF length, are paramount to ensure sufficient flap coverage of the surgical corridor.

As previously mentioned, CSF leaks are a major concern of all endoscopic endonasal procedures. In a case series of 55 pediatric patients, $67 \%$ of surgeries had intraoperative CSF leaks, and $23 \%$ had postoperative leaks. ${ }^{9}$ The use of vascularized versus nonvascularized flaps did not contribute to postoperative CSF leak, and site or tumor pathology was not a predictive factor. Age (5.8-9.6 years and $>16.2$ years) and clival chordomas were the only high-risk predictors found. ${ }^{5}$ The 11 patients aged 5.7 years and below did not experience postoperative CSF leaks in this study. Low rates of postoperative CSF leaks have been reported in other case series as well, and they can effectively be repaired by an endonasal approach. ${ }^{13,14}$ Stapleton et al also found that postoperative hydrocephalus and lumbar drains were not risk factors for CSF, and that lumbar drains are not indicated for intraoperative CSF leaks, despite the call for its use as initial management of postoperative CSF leaks. ${ }^{9,10}$ A recent randomized trial at UPMC in adults has shown that lumbar drains do play a role in transclival and large anterior approaches, but not in sellar or suprasellar defects. ${ }^{9}$ None 


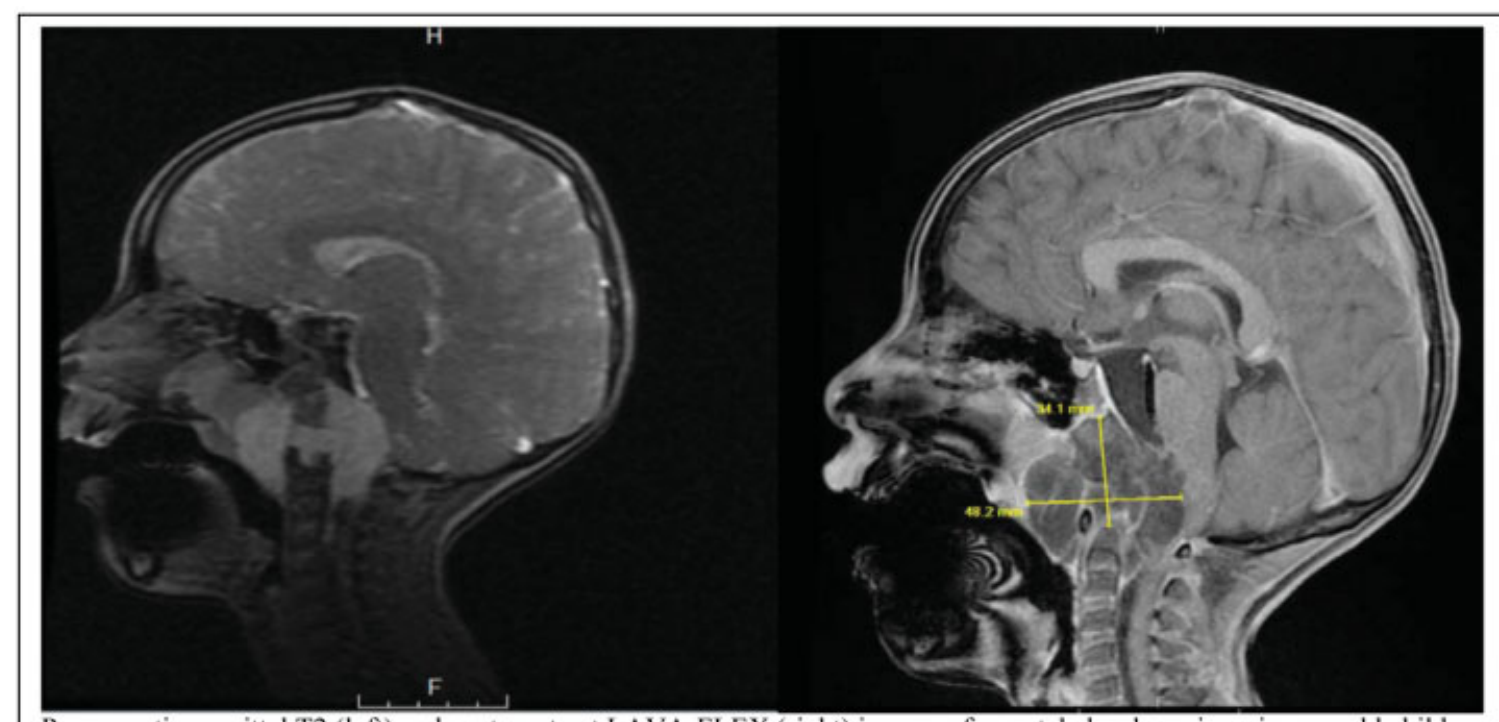

Pre-operative sagittal T2 (left) and post-contrast LAVA-FLEX (right) images of a septal chordoma in a six year old child. The tumor had caused $\mathrm{Cl}$ vertebral body destruction, compressed the cervical medullary junction at the level of the foramen magnum, displaced the left vertebral artery, and was compressing the nasopharynx, adenoids and soft palate. Patient received a transsellar/transclival resection, requiring a double nasoseptal flap.

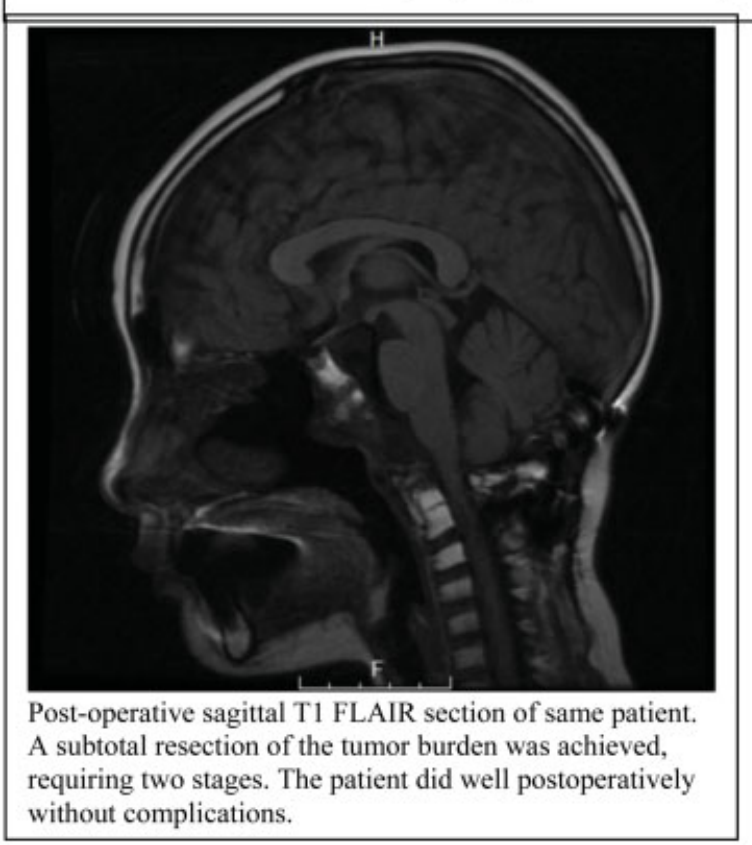

Fig. 2 Pre- and postoperative magnetic resonance imaging, Patient 7. (A) Preoperative sagittal T2 (left) and postcontrast LAVA-FLEX (right) images of a septal chordoma in a 6-year-old child. The tumor had caused C1 vertebral body destruction, compressed the cervical medullary junction at the level of the foramen magnum, displaced the left vertebral artery, and was compressing the nasopharynx, adenoids and soft palate. Patient received a transsellar/transclival resection, requiring a double nasoseptal flap. (B) Postoperative sagittal T1 fluid-attenuated inversion recovery section of same patient. A subtotal resection of the tumor burden was achieved, requiring two stages. The patient did well postoperatively without complications.

of our patients received lumbar drains or experienced postoperative CSF leaks, but further studies are warranted to assess the role for lumbar drains in this young pediatric population.

For the first time to our knowledge, this study focuses on the utility of EEA in the young pediatric population aged 6 years and under. We feature eight children with a wide array of skull base tumors, with broad pathology and surgical technique. NSF was utilized in several of the patients, with various methods of surgical corridor repair. GTR was successful in the majority of cases, and only the two complex chordoma cases required staged resections. Only one patient had residual endocrine sequalae, and outcomes overall were excellent, with the exception of one patient who died due to metastatic disease of their neuroblastoma. All patients followed up in clinic, with average follow-up of over 17 months. However, this study is limited due to its small sample size of only eight patients. While having a variety of tumor 
pathologies is important for the assessment of EEA, we are unable to form conclusions on the approach for specific tumor types, as each tumor was only seen once in this series, with the exception of the two chordoma cases. Additionally, due to our limited number of patients, we were unable to determine any generalizable conclusion of the use of NSF in this population, as only three patients received NSF. Large studies are needed to look specifically at this technique in this young population. While our extended follow-up may be viewed as a potential strength of this study, there may be future complications that were not captured in the 1 to 2 years follow-up period. The literature has highlighted potential delayed complications, such as pontine herniation following a transclival approach, ${ }^{15}$ which may be captured with a longer study follow-up period. However, many complications attributed to EEA such as pontine herniation have been shown in the adult population, and limited data have supported translational results in young children. Studies with longer follow-up periods are needed for long-term complication surveillance, and specifically in the NSF subpopulation, to confirm long-term flap adherence.

\section{Conclusion}

In this case series, the pediatric endoscopic endonasal approach was found to be safe and effective for the resection of a variety of skull base tumors in patients aged 6 and under. Despite the developmental anatomical challenges presented in younger children, our series supports the use of EEA in similar manners as performed in older children, including the utilization of typical approaches such as NSFs. Larger scale studies are warranted to fully evaluate the efficacy of this approach in the young pediatric patient population for the variety of tumor pathologies, and to compare this population with the established literature on older pediatric patients and adults.

\section{References}

1 Kassam A, Thomas AJ, Snyderman C, et al. Fully endoscopic expanded endonasal approach treating skull base lesions in pediatric patients. J Neurosurg 2007;106(2, Suppl):75-86
2 Shah RN, Surowitz JB, Patel MR, et al. Endoscopic pedicled nasoseptal flap reconstruction for pediatric skull base defects. Laryngoscope 2009;119(06):1067-1075

3 Pirris SM, Pollack IF, Snyderman CH, et al. Corridor surgery: the current paradigm for skull base surgery. Childs Nerv Syst 2007;23 (04):377-384

4 Castelnuovo P, Bignami M, Pistochini A, Battaglia P, Locatelli D, Dallan I. Endoscopic endonasal management of encephaloceles in children: an eight-year experience. Int J Pediatr Otorhinolaryngol 2009;73(08):1132-1136

5 Locatelli D, Rampa F, Acchiardi I, Bignami M, Pistochini A, Castelnuovo P. Endoscopic endonasal approaches to anterior skull base defects in pediatric patients. Childs Nerv Syst 2006;22(11): $1411-1418$

6 Jeswani S, Nuño M, Wu A, et al. Comparative analysis of outcomes following craniotomy and expanded endoscopic endonasal transsphenoidal resection of craniopharyngioma and related tumors: a single-institution study. J Neurosurg 2016;124(03):627-638

7 Ceylan S, Koc K, Anik I. Endoscopic endonasal transsphenoidal approach for pituitary adenomas invading the cavernous sinus. J Neurosurg 2010;112(01):99-107

8 Tatreau JR, Patel MR, Shah RN, et al. Anatomical considerations for endoscopic endonasal skull base surgery in pediatric patients. Laryngoscope 2010;120(09):1730-1737

9 Stapleton AL, Tyler-Kabara EC, Gardner PA, Snyderman CH, Wang EW. Risk factors for cerebrospinal fluid leak in pediatric patients undergoing endoscopic endonasal skull base surgery. Int J Pediatr Otorhinolaryngol 2017;93:163-166

10 Hamid O, El Fiky L, Hassan O, Kotb A, El Fiky S. Anatomic variations of the sphenoid sinus and their impact on trans-sphenoid pituitary surgery. Skull Base 2008;18(01):9-15

11 Purcell PL, Shinn JR, Otto RK, Davis GE, Parikh SR. Nasoseptal flap reconstruction of pediatric sellar defects: a radiographic feasibility study and case series. Otolaryngol Head Neck Surg 2015; 152(04):746-751

12 Ghosh A, Hatten K, Learned KO, et al. Pediatric nasoseptal flap reconstruction for suprasellar approaches. Laryngoscope 2015; 125(11):2451-2456

13 Giannoni CM, Whitehead WE. Use of endoscopic vascularized nasoseptal flap in children. Otolaryngol Head Neck Surg 2013;148 (02):344-346

14 Emanuelli E, Bossolesi P, Borsetto D, D’Avella E. Endoscopic repair of cerebrospinal fluid leak in paediatric patients. Int J Pediatr Otorhinolaryngol 2014;78(11):1898-1902

15 Koutourousiou M, Filho FV, Costacou T, et al. Pontine encephalocele and abnormalities of the posterior fossa following transclival endoscopic endonasal surgery. J Neurosurg 2014;121(02): 359-366 